\title{
EFFECT OF HIGH PRESSURE COOLANT JET ON CUTTING TEMPERATURE, TOOL WEAR AND SURFACE FINISH IN TURNING HARDENED (HRC 48) STEEL
}

\author{
Mozammel Mia ${ }^{1}$ and Nikhil Ranjan Dhar ${ }^{2 *}$ \\ ${ }^{1}$ Department Mechanical and Production Engineering \\ Ahsanullah University of Science and Technology, Dhaka-1208, Bangladesh \\ ${ }^{2}$ Department of Industrial and Production Engineering \\ Bangladesh University of Engineering and Technology, Dhaka-1000, Bangladesh \\ *Corresponding e-mail: nrdhar@ipe.buet.ac.bd
}

\begin{abstract}
Hard turning of harder material differs from conventional turning because of its larger specific cutting forces requirements. The beneficial effects of hard turning can be offset by excessive temperature generation which causes rapid tool wear or premature tool failure if the brittle cutting tools required for hard turning are not used properly. Under these considerations, the concept of high-pressure coolant (HPC) presents itself as a possible solution for high speed machining in achieving slow tool wear while maintaining cutting forces at reasonable levels, if the high pressure cooling parameters can be strategically tuned. This paper deals with an experimental investigation of some aspects of the turning process applied on hardened steel (HRC48) using coated carbide tool under high-pressure coolant, comparing it with dry cut. The results indicate that the use of high-pressure coolant leads to reduced surface roughness, delayed tool flank wear, and lower cutting temperature, while also having a minimal effect on the cutting forces.
\end{abstract}

Key Words: Hardened steel, HPC, Cutting Temperature, Tool Wear and Surface Finish

\section{INTRODUCTION}

Hardened steel is frequently used in the automotive industry to manufacture bearings, gears, shafts and cams requiring tight geometric tolerances, longer service life and good surface finish ${ }^{1,2}$. The conventional approach for the production of these parts involves a sequence of forming, annealing, rough cutting and heat treatment followed by a final grinding operation to obtain the desired surface roughness. Two procedures from the conventional processes can be eliminated by applying Hard Turning process, that is, annealing and grinding ${ }^{3}$. But machining of hardened steel is a very complex phenomenon which results in poor surface finish, high tool wear, premature failure of tools etc.

There are several causes those are directly and indirectly related to poor surface finish of the product and short cutting tool life among which generation of huge amount of heat at the chip-tool interface during hard turning is most prominent. The elevated temperature softens and weakens the tool; the tool tip become blunt and in result, produce poor surface finish, dimensional deviation, surface and subsurface defects including micro-cracks and shorten the tool life. As a result, it's important to investigate the work piece-tool behavior under different machining environments.

Cutting fluid and its method of application should be so chosen that it will not react at the interface zone but will form a low shear strength solid lubricant. Machining without the cutting fluid would be most appropriate if the cooling and friction reduction were not necessary. To address environmental, health, economic, and performance challenges, it is essential to develop and implement appropriate lubrication process. Some of the alternative approaches are using biodegradable and cryogenic coolants ${ }^{4-7}$.

An emerging cutting fluid application technology to control the cutting temperature is High Pressure Coolant (HPC) method. High pressure coolant injection technique not only provided the reduction in cutting force and temperature but also reduced the consumption of cutting fluid by $50 \%{ }^{9}$. It reduces the chip-tool contact area, minimizes friction at the chiptool interface, and limits heat transfer to the cutting tool and consequently improving tool performance during machining ${ }^{10}$. Machining of alloys at high speed conditions can be achieved by a combination of the appropriate tool material, machining technique and the choice of a suitable cooling technology ${ }^{11}$. The credibility of high-pressure coolant assisted machining had been thoroughly investigated over the years ${ }^{11-14}$. The system not only provides adequate cooling at the tool-workpiece interface but also provides an effective removal (flushing) of chips from the cutting area. Excellent chip breakability has been reported when machining difficult-to-cut materials with high-pressure coolant supply.

At low cutting speed, almost four times longer tool life is obtained by the proper cutting fluid. Ezugwu and Bonney ${ }^{13}$ reported that machining Inconel-718 with coated carbide inserts under highpressure coolant supplies improve tool life by up to 7 folds.

Several studies have investigated the capability of the process to compete with grinding, and most have concluded that at proper conditions with a good machine, hard turning can produce dimensional accuracy and surface finishes acceptable for most applications.

The application of HPC over conventional fluid in hard turning may be beneficial in terms of surface 
finish, tool wear, tool life, cutting zone temperature etc. The overall success of implementing HPC in the realm of metal removal industries therefore depends on increased research activities providing credible data for sound understanding of high pressure coolant supplies at the chip-tool interface and integrity of hard turned machined components. In this regard, the present research work is carried out to experimentally investigate the role of highpressure coolant jet in machining hardened steel (48 HRC) by coated carbide insert at different speed and feed combinations in respects of chip reduction coefficient, average chip-tool interface temperature, tool wear, and surface roughness.

\section{EXPERIMENTAL CONDITIONS}

In this present research work, intensive experimental investigations have been carried out in turning hardened steel (48 HRC) by coated carbide insert (SNMG). Investigations have incorporated close observations of the cutting temperature as a response which act as a function of turning operations. Cutting fluids are widely used to reduce the temperature. But due to corrosion and contamination of natural environment, high pressure coolant jet machining has been implemented here in order to have better experimental results.

The material used in the research was hardened medium carbon steel (HRC 48) with approximately $0.48 \%$ carbon content. It was a hollow cylindrical bar of length $200 \mathrm{~mm}$ with external and internal diameters of $120 \mathrm{~mm}$ and $45 \mathrm{~mm}$ respectively as shown in Fig.1.

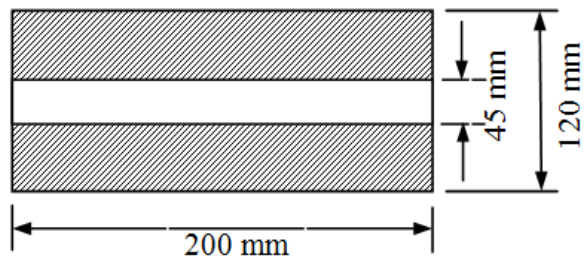

Figure 1. Work material specimen for hardening.

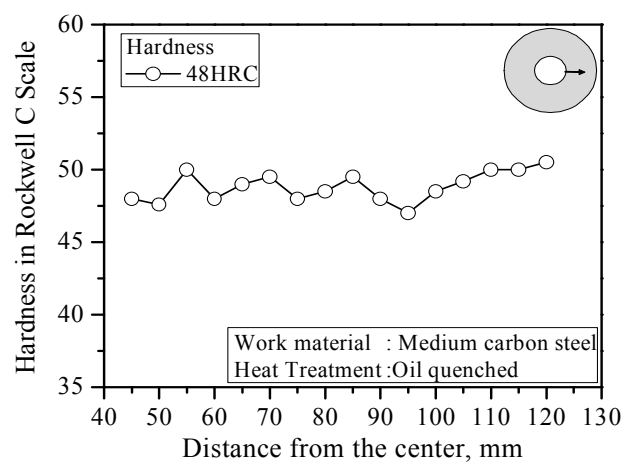

Figure 2. Variation of hardness along the radius.

The electric furnace of a high heating element $\left(\mathrm{RG}-3000^{\circ} \mathrm{C}\right)$ was used for heat treatment. It took three hours to raise the temperature to $900^{\circ} \mathrm{C}$ and soaked the work material at that temperature for one and half hour in the heating chamber. A quench tank having (Bluta oil grade 27) capacity 600 liters was used for quenching the work material. The oil reduced the absorption of atmospheric gasses that, in turn reduced the amount of bubbles. As a result, oil wet the metal surface and cooled it more rapidly than water.

Quenched medium carbon steel always required to temper because steel is often harder than needed and too brittle for most practical uses. It was done by heating the workpiece to a specific temperature $\left(300^{\circ} \mathrm{C}\right)$, holding it at that temperature for two hour and then cooling it instill air. The hardness distribution within the sample is shown in Fig.2.

The machining tests were carried out by straight turning of hardened steel (48 HRC) in a reasonably rigid and powered centre lathe $(7.5 \mathrm{~kW}$, China) at different cutting speeds $\left(\mathrm{V}_{\mathrm{c}}\right)$ and feed rates $\left(\mathrm{S}_{\mathrm{o}}\right)$ under both dry and high-pressure coolant conditions. Keeping in view less significant role of the depth of cut $(t)$ on cutting temperature, the depth of cut was kept fixed to only $1.0 \mathrm{~mm}$. But in the tool wear investigation the depth of cut was taken $2.0 \mathrm{~mm}$.

The tool geometry is reasonably expected to play significant role on such cooling effectiveness. Keeping these views, the tool configuration SNMG and tool holder PSBNR (Widia) type have been undertaken for the present investigation. The positioning of the nozzle tip with respect to the cutting insert has been settled after a number of trials. The high pressure coolant jet is directed along the auxiliary cutting edge at an angle $20^{\circ}$ to reach at the principal flank and partially under the flowing chips through the in-built groove parallel to the cutting edges. The photographic view of the experimental set-up is shown in Fig.3.

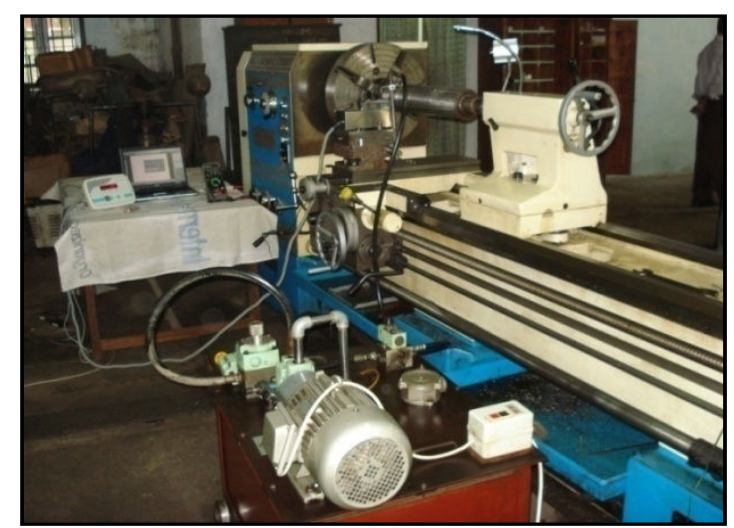

Figure 3. Photographic view of experimental set-up.

The ranges of cutting speed and feed rate chosen in the present investigation are representative of the current industrial practice for the tool-work material combination that has been investigated. The conditions under which the machining tests have been carried out are briefly given in Table 1 . The machining responses have been monitored and studied using sophisticated and reliable equipment and techniques as far as possible. 
Table 1. Experimental conditions

\begin{tabular}{|ll|}
\hline Machine tool & $:$ Lathe (China), $7.5 \mathrm{~kW}$ \\
Work materials & $:$ Hardened medium carbon steel \\
Hardness & $: 48 \mathrm{HRC}$ \\
Cutting tool & $:$ SNMG 120408, Widia \\
Coating & $:$ TiCN, WC, Co \\
Geometry & $:-6^{\circ}, 6^{\circ}, 6^{\circ}, 6^{\circ}, 15^{\circ}, 75^{\circ}, 0.8(\mathrm{~mm})$ \\
Tool holder & $:$ PSBNR $2525 \mathrm{M} 12, \mathrm{Widia}$ \\
Process parameters & \\
Cutting speed & $: 58,81,115$ and $161 \mathrm{~m} / \mathrm{min}$ \\
Feed rate & $: 0.10,0.12,0.14$ and $0.16 \mathrm{~mm} / \mathrm{rev}$ \\
Depth of cut & $: 1.0 \mathrm{~mm}$ and $2.0 \mathrm{~mm}$ \\
High pressure $: 80$ bar, Coolant: $6.0 \quad \mathrm{~L} / \mathrm{min}$ \\
coolant & through external nozzle \\
Coolant type & $:$ VG-68 (ISO grade) \\
Environment & $:$ Dry and HPC condition \\
\hline
\end{tabular}

The thickness of the chips was repeatedly measured by a slide caliper to determine the value of the chip reduction coefficient. The average cutting temperature was measured by simple but reliable tool-work thermocouple technique with proper calibration. The life of the tool is generally assessed by the average value of the principal flank wear $\left(\mathrm{V}_{\mathrm{B}}\right)$. During machining under each condition, the cutting insert was withdrawn at regular intervals and $V_{B}$ was measured under an optical microscope (Carl Zeiss, Germany) fitted with a precision micrometer. The pattern and extent of wear of the tool tip have been observed under Scanning Electron Microscope (Philips XL 30, Belgium). After machining by SNMG insert, at different $\mathrm{V}_{\mathrm{c}}-\mathrm{S}_{\mathrm{o}}$ combinations under both dry and HPC conditions the surface roughness was measured by a Talysurf (Surtronic $3+$ ).

\section{EXPERIMENTAL RESULTS}

An important machinability index is chip reduction coefficient, $\xi$ (ratio of chip thickness after and before cut). The variation in value of chip reduction coefficient with the change in cutting speeds and feed rates as well as machining environment evaluated for hardened medium carbon steel has been plotted and shown in Fig.4.

High production machining associated with high velocity and feed rate inherently generates high heat as well as high cutting zone temperature. In a normal cutting condition, all such heat sources produce maximum temperature at the chip-tool interface, which substantially influence the chip formation mode; tool wear pattern, tool life and product quality.

The evaluated role of HPC on average chip-tool interface temperature for all $\mathrm{V}_{\mathrm{c}}-\mathrm{S}_{0}$ combinations has been shown in Fig. 5 .

Tool wear initially starts with a relatively faster rate due to what is called break-in wear caused by attrition and micro-chipping at the sharp cutting edges. Cutting tools may also often fail prematurely, randomly and catastrophically by mechanical breakage and plastic deformation under adverse machining conditions. However, in the present investigations with the tool and work material and the machining conditions undertaken, the tool failure mode has been mostly gradual wear. The growth of average flank wear, $V_{B}$ with the progress of machining time recorded while turning HRC 48 hardened steel by SNMG insert under both dry and HPC conditions has been shown in Fig. 6.

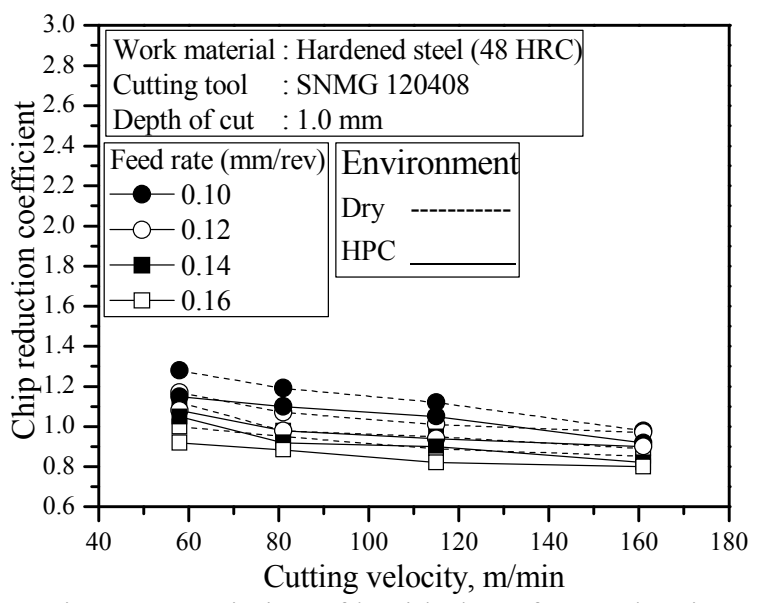

Figure 4. Variation of $\xi$ with that of $\mathrm{V}_{\mathrm{c}}$ and $\mathrm{S}_{\mathrm{o}}$ in turning hardened steel under dry and HPC conditions.

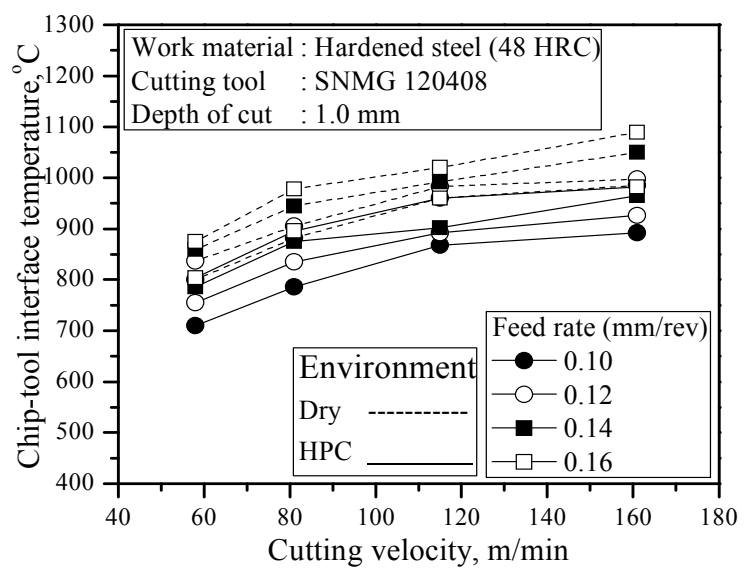

Figure 5. Variation of cutting temperature with that of $\mathrm{V}_{\mathrm{c}}$ and $\mathrm{S}_{\mathrm{o}}$ in turning hardened steel under dry and HPC conditions.

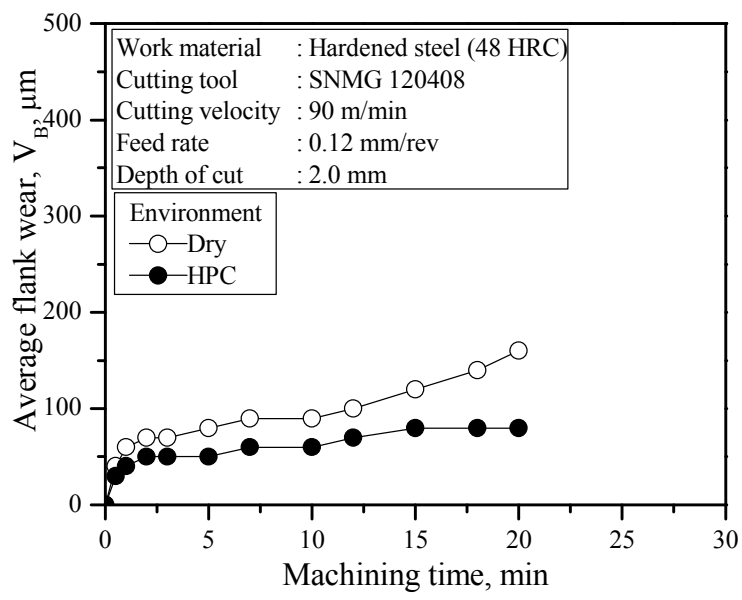

Figure 6. Growth of $\mathrm{V}_{\mathrm{B}}$ in SNMG insert during turning hardened steel under dry and HPC conditions. 
Figure 7 shows the scanning electron microscopy (SEM) views of the worn out insert (SNMG) after being used for machining hardened medium carbon steel of hardness $48 \mathrm{HRC}$ at $\mathrm{V}_{\mathrm{c}}=90 \mathrm{~m} / \mathrm{min}, \mathrm{S}_{\mathrm{o}}=0.12$ $\mathrm{mm} / \mathrm{rev}$ and $\mathrm{t}=2.0 \mathrm{~mm}$ for $20 \mathrm{~min}$. under dry and high pressure coolant condition.

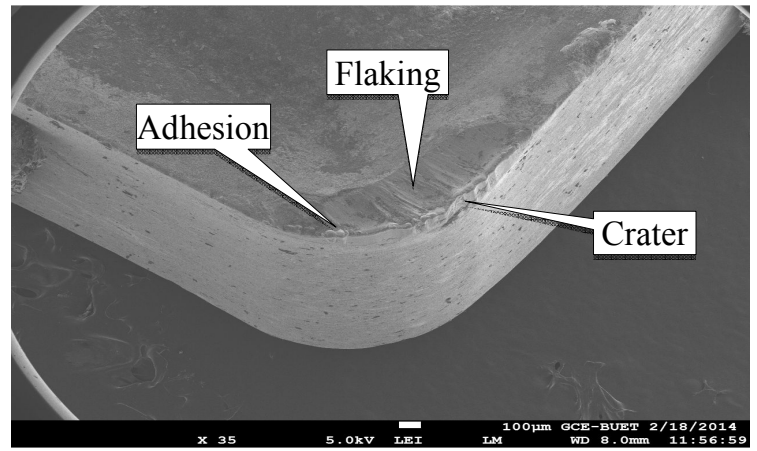

(a) Dry machining (20 min)

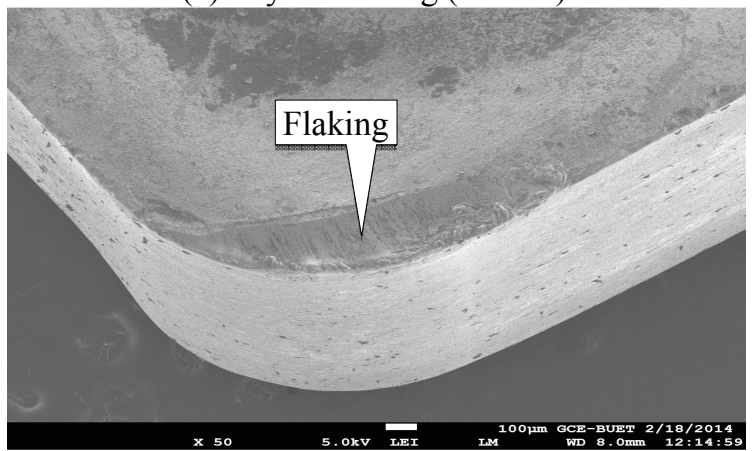

(b) HPC machining (20 min)

Figure 7. SEM views of the worn out SNMG insert after machining hardened steel under (a) dry and (b) HPC conditions.

Surface roughness is an important index of machinability which is substantially influenced by the machining environment for a given tool-work pair and speed-feed condition. Surface roughness has been measured at two stages; first, after a few seconds of machining with the sharp tool while recording the cutting temperature and second, with the progress of machining while monitoring growth of tool wear with machining time. The surface roughness attained after 50 seconds of machining is shown in Fig.8.

The variation in surface roughness in machining hardened medium carbon steel by SNMG insert observed with the progress of machining time has been shown in Fig.9.

\section{DISCUSSION ON RESULTS}

Chip properties largely depend on different hardness values of the work materials; tool geometry-especially the rake angle and the cutting parameters i.e. cutting speed, feed rate and depth of cut.

They are also somewhat dependent on the cutting environments and the nature of the chip-tool interaction. Fig. 4 reveals that most of the values of chip reduction coefficient $(\xi)$ are more than 1.0, and
HPC produces lower values of $\xi$. It is because of the groove present on the rake surface of the SNMG insert that restrict the expansion of the chips under compression which reduces the cut chip thickness. As the cutting speed and feed rate increase, the value of the chip reduction coefficient decreases because of the higher energy consumption associated with the higher material removal rate.

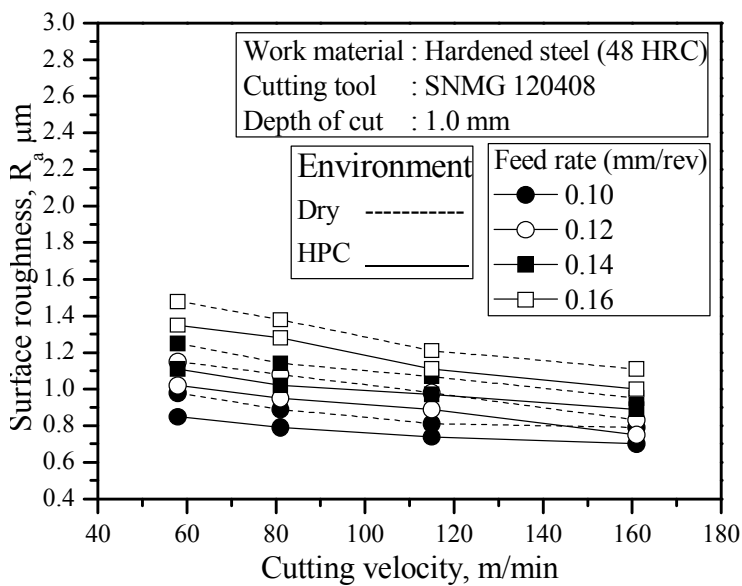

Figure 8. Variation in $\mathrm{R}_{\mathrm{a}}$ observed after turning hardened steel by SNMG insert at different $V_{c}$ and $\mathrm{S}_{\mathrm{o}}$ under dry and HPC conditions.

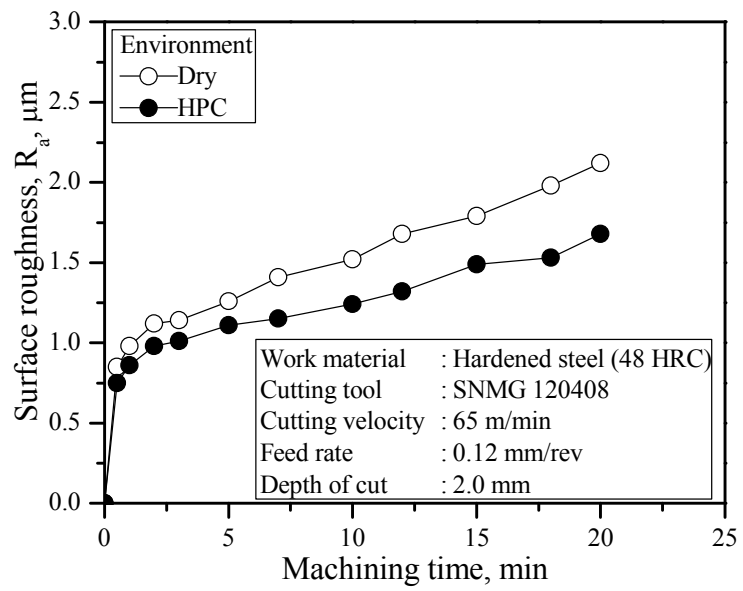

Figure 9. Surface roughness developed with progress of turning hardened steel by SNMG insert under dry and HPC conditions.

During machining, shearing of work material, friction between the flowing chips and rake face of the tool and friction of auxiliary flank with finished surface are the principal sources of heat generation. The magnitude of the cutting temperature increases with the increase of material removal rate i.e. with the increase of cutting velocity, feed and depth of cut in hard turning of different hardened steels, as a result, high production machining is constrained by the ascend in temperature.

The evaluated role of HPC on average chip-tool interface temperature in turning hardened steel at different $\mathrm{V}_{\mathrm{c}}-\mathrm{S}_{0}$ combinations in compare to the dry condition has been shown in Fig.5. At high speedfeed conditions, the MRR is high which requires high energy consumption. This high energy input helps 
the cutting zone temperature to increase. This is why the controlling of temperature is more important in high speed machining.

From Fig.5 it is evident that with the increase in cutting velocity, the cutting temperature increases for both dry and HPC conditions but the cooling effect gets more significant at higher speeds where the temperature difference between dry machining and HPC machining is higher. The possible reason may be that high pressure coolant jet reached the chip-tool interface even at high speed-feed condition. The high-velocity jet uplifted the chip creating a wedge effect and reached at the shear zone. There it performed both the cooling and lubricating effects and eventually reduced the temperature. This reduction in temperature is very much appreciable in retaining tool life and product quality.

At conventional speed, tool wear is mainly due to abrasion and adhesion but is dominated by diffusion process at higher speeds. High pressure coolant directly reduces the diffusion process by decreasing the cutting zone temperature. As a result, a delayed progression of tool wear is followed under the high pressure coolant condition and no crater wear is formed as exhibited at the SEM views shown in Fig.7. This promotes longer tool life and reduces tool break. Average principal flank wear, $\mathrm{V}_{\mathrm{B}}$ is considered to be the most significant parameter at least in research work. Fig.6 shows the growth of average principal flank wear $\left(\mathrm{V}_{\mathrm{B}}\right)$ with time for dry and HPC environments with SNMG insert for HRC 48 hardened steel. It is obvious from the figure that for a certain period of time the magnitude of $V_{B}$ is lower in case of HPC assisted machining.

In the high pressure cooling system, the coolant jet was pressurized about 80 bars. This high pressure carried the coolant jet into the plastic contact between tool tip and workpiece where it cooled the interface and lubricated properly. It also helped to reduce the heat generation caused by the friction between auxiliary flank face and finished surface by appropriate lubrication. Such improvement by high pressure coolant may also be attributed to retention of proper sharpness and hardness of the cutting edge by the steady and intensive cooling, protection from oxidation and corrosion and absence of built up edge formation which accelerates both crater wear and flank wear by flaking and chipping.

The surface roughness attained after 50 seconds of machining of the hardened steel by the sharp SNMG insert at various $\mathrm{V}_{\mathrm{c}}-\mathrm{S}_{\mathrm{o}}$ combinations under dry and HPC conditions are shown in Fig.8. Reduction of cutting temperature and average principal tool wear due to the application of high pressure coolant facilitate smooth machined surface. The lubrication provided by the pressurized oil at the chip-tool contact point prevent rubbing of tool and chip on the surface that eventually reduces the surface roughness. Prevention of BUE formation on the insert due to the application of HPC is also responsible for the production better surface finish.
Fig.9 shows the development of $\mathrm{R}_{\mathrm{a}}$ with progression of machining time for cutting velocity $65 \mathrm{~m} / \mathrm{min}$, feed rate $0.12 \mathrm{~mm} / \mathrm{rev}$ and depth of cut $1.5 \mathrm{~mm}$ for hardness values of HRC 48. Two fresh inserts of SNMG are used separately for dry and HPC conditions along with tool wear measurement. The HPC assisted machining reduces the surface roughness. The aforesaid reasons are also responsible to reduce the surface roughness.

\section{CONCLUSIONS}

Based on the observation made and the experimental results obtained, the following conclusions are made:

(i) Chip-tool interface temperature increases with the increase in cutting velocity and feed rate. The application of high pressure coolant provides a substantial reduction in cutting temperature.

(ii) The present high pressure coolant system enabled the reduction in average chip-tool interface temperature up to $12 \%$ for SNMG insert depending on the cutting conditions.

(iii) Application of high pressure coolant jet produced reduced tool wear, good dimensional accuracy and surface finishes that are acceptable for most applications. In general, this study shows that hard turning is capable of producing precision parts and can replace grinding for many applications.

(iv) Tool wear is proportional to surface roughness for any specified tool-workpiece. Application of HPC reduces both the parameters but their relation remains unaltered.

\section{ACKNOWLEDGEMENT}

The authors would like to acknowledge the support of DAERS, BUET for the permission to perform the research in the central machine shop, BUET.

\section{REFERENCES}

1.G. Dawson and R. Kurfess, 2001 "Tool Life, Wear Rates, and Surface Quality in Hard Turning", Transaction of North American Manufacturing Research Inst SME, pp. 175-182.

2. J. Kundrak, B. Karpuschewski, and K. Gyani, 2008 "Accuracy of Hard Turning”, J. of Mat. Processing Technology, Vol. 202, pp. 328-338.

3. W. Grzesik, 2011 Mechanics of Cutting and Chip Formation. In: Davim JP (ed.), "Machining of Hard Materials", London: Sprgr., pp. 87-114.

4. O. Cakir, A. Yardimen, T. Ozben, and E. Kilickap, 2007 "Selection of Cutting Fluids in Machining Process", J. of Achievements in Mat. And Manf. Engineering, Vol. 25(2), pp. 99-102.

5. N. R. Dhar, and M. Kamruzzaman, 2007 "Cutting Temperature, Tool Wear, Surface Roughness and Dimensional Deviation in Turning AISI-4037 Steel 
under Cryogenic Condition”, International Journal of Machine Tool and Manufacture, Vol.47(5), pp.754-759.

6. W. Grzesik, and K. Zak, 2014 "Characterization of Surface Integrity Produced by Sequential Dry Hard Turning and Ball Furnishing Operations", J of Manf. Sci. and Eng., Vol. 136, pp. 34-39.

7. Y. M. Sashidhara and S. R. Jayaram, 2010 "Vegetable Oils as a Potential Cutting Fluid-An Evolution”, Tri. Inter., Vol. 43, pp. 1073-1081.

8. N. R. Dhar, S. Islam, and M. Kamruzzaman, 2007 "Effect of Minimum Quantity Lubrication (MQL) Tool Wear and Surface Roughness and Dimensional Deviation in Turning AISI-4340 Steel", G.U. Journal of Science, Vol. 20 (2), pp. 23-32, January-June.

9. D. Umbrella, and L. Filice, 2009 "Improving Surface Integrity in Orthogonal Machining of Hardened AISI 52100 Steel by Modeling White and Dark Layers Formation", CIRR, Vol. 58, pp. 73-76.

10. E. O. Ezugwu, J. Bonney, and M. H. Abu Bakar, 2011 "Effect of Coolant Properties on Tool Life During Machining of Ti-6Al-4V Alloy Under High Pressure Coolant Supplies", Proceedings of Int. Conf. of the Soc. of Tribologists and Lubri. Eng., Atlanta, Georgia, USA, 15-19 May.
11. E. O. Ezugwu, J. Bonney, and Y. Yamane, 2003 "An Overview of the Machinability of Aeroengine Alloys", Journal of Materials Processing Technology, Vol. 134, pp. 233-253.

12. E. O. Ezugwu, R. B. Da Silvaa, J. Bonney, and A. R. Machado, 2005 "Evaluation of the Performance of CBN Tools when Turning Ti-6Al4V Alloy with High Pressure Coolant Supplies", International Journal of Machine Tools \& Manufacture, Vol. 45, pp. 1009-1014.

13. E. O. Ezugwu, and J. Bonney, 2004 "Effect of High-Pressure Coolant Supply when Machining Nickel-Base, Inconel 718, Alloy with Coated Carbide Tools", Journal of Materials Processing Technology, Vol. 153-154, pp. 1045-1050.

14. R. B. Silva, A. R. Machado, E. O. Ezugwu, J. Bonney, and W. F. Sales, 2013 "Tool Life and Wear Mechanisms in High Speed Machining of Ti-6Al-4V Alloy with PCD Tools Under Various Coolant Pressures", J. of Material Processing Tech., Vol. 213, pp. 1459-1464. 